\title{
RELACIÓN ENTRE REPRESENTACIÓN DE ENFERMEDAD, REPRESENTACIÓN DEL TRATAMIENTO Y ADHERENCIA EN ADULTOS CON ASMA: UNA REVISIÓN
}

\author{
Isaías Vicente Lugo González y Cynthia Zaira Vega Valero \\ Universidad Nacional Autónoma de México \\ México
}

\begin{abstract}
RESUMEN
La adherencia al medicamento de control es el pilar fundamental para el control del asma; sin embargo, se ha identificado que una representación negativa de la enfermedad y el tratamiento impactan de modo negativo en esta. Se hizo una revisión narrativa para identificar los estudios empíricos acerca de representación de enfermedad, representación del tratamiento y adherencia a medicamentos de control en adultos con asma, desde el modelo de sentido común y representación de la enfermedad (MSCRE). Se identificaron 17 estudios divididos en transversales y longitudinales (12), experimentales (3) y de intervención (2), reportando que las principales dimensiones del MSCRE asociadas con la adherencia son la necesidad del tratamiento, preocupación, control de la enfermedad y del tratamiento, temporalidad crónica y consecuencias. Se concluye que el MSCRE es un modelo psicológico que tiene aplicaciones en la investigación y atención clínica para explicar y promover conductas de adherencia a medicamentos de control en adultos con asma.
\end{abstract}

Palabras Clave:

representación de enfermedad; representación del tratamiento; adherencia, adultos, asma

\author{
RELATIONSHIP BETWEEN ILLNESS REPRESENTATION, TREATMENT \\ REPRESENTATION AND ADHERENCE IN ADULTS WITH ASTHMA: A REVIEW
}

\begin{abstract}
Adherence to control medication is the fundamental pillar for the control of asthma, however, it has identified that a negative illness representation and the treatment have a negative impact on it. A narrative review was conducted to identify the empirical studies on illness representation, treatment representation and adherence to control medication in adults with asthma, from the Common-Sense Model and IIIness Representation (CSM). We identified 17 studies divided into cross-sectional and longitudinal (12), experimental (3) and intervention (2), reporting that the main dimensions of the CSM linked with adherence are the necessity for treatment, concern, illness control and treatment, chronic temporality and consequences. It is concluded that the CSM is a psychological model that has applications in clinical research and attention to explain and promote adherence behaviors to control medications in adults with asthma.
\end{abstract}

Keywords:

illness representation; treatment representation; adherence; adults; asthma.

Bitácora del Artículo:

| Recibido: 26 de Diciembre de 2018 | Aceptado: 10 de Septiembre de 2019 | Publicado en línea: Enero - Junio de 2020 | 


\title{
RELACIÓN ENTRE REPRESENTACIÓN DE ENFERMEDAD, REPRESENTACIÓN DEL TRATAMIENTO Y ADHERENCIA EN ADULTOS CON ASMA: UNA REVISIÓN
}

\author{
Isaías Vicente Lugo González y Cynthia Zaira Vega Valero \\ Universidad Nacional Autónoma de México \\ México
}

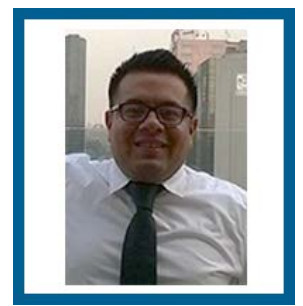

Isaías Vicente Lugo González

FES- Iztacala, UNAM

Correo: isaiasvlg@comunidad.unam.mx

Estudiante del doctorado en Psicología y salud, Facultad de Estudios Superiores Iztacala. UNAM. Docente de posgrado en psicología. Facultad de Estudios Superiores Iztacala. UNAM. Docente de posgrado de la Universidad del Distrito Federal, Campus Santa María.

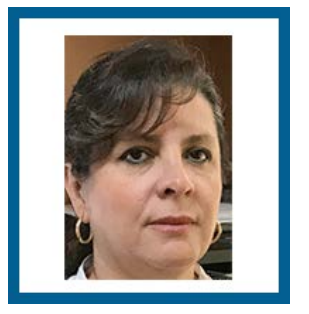

Cynthia Zaira Vega Valero

FES- Iztacala, UNAM

Correo: vegavalero@hotmail.com

Doctora en Psicología por la UNAM, Profesora Titular de la Carrera de Psicología y Coordinadora de la Residencia en Gestión Organizacional de la Maestría en Psicología. de la FES Iztacala, Universidad Nacional Autónoma de México.

\section{CONTRIBUCIÓN DE LOS AUtORES}

Isaías Vicente Lugo González: Concibió la idea del proyecto, realizó la búsqueda de la bibliografía en bases de datos y redactó del manuscrito. I Cynthia Zaira Vega Valero: concibió la idea del proyecto , realizó larevisión y supervisión del manuscrito.

\section{AGRADECIMIENTOS}

Agradecemos a todas aquellas personas que participaron e hicieron posible la realización de este estudio.

\section{DATOS DE FiLIACIÓN DE LOS AUtORES}

Facultad de Estudios Superiores Iztacala, UNAM

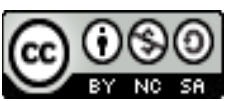

Copyright: ( 2020 Lugo-González, I.V. \& Vega-Valero, C.

Este es un artículo de acceso abierto distribuido bajo los términos de la licencia Creative Commons Reconocimiento-NoComercial 4.0 Internacional, por lo que su contenido gráfico y escrito se puede compartir, copiar y redistribuir total o parcialmente sin necesidad de permiso expreso de sus autoras con la única condición de que no se puede usar con fines directamente comerciales y los términos legales de cualquier trabajo derivado deben ser los mismos que se expresan en la presente declaración. La única condición es que se cite la fuente con referencia a la Revista Digital Internacional de Psicología y Ciencia Social y a sus autoras. 


\section{TABLA DE CONTENIDO}

Objetivo, 37

MÉTODO

Rango de la búsqueda, 37

Unidades de análisis, 37

Núcleos temáticos, 37

Artículos encontrados, 38

Composición de la muestra de artículos, 38

Criterio de inclusión y exclusión de artículos, 38

Procedimiento, 38

\section{RESULTADOS}

Características de los estudios, 39

Características de la muestra, 39

Relación y predicción de las variables, 39

Estudios experimentales, 41

Características de la muestra, 41

Relación y predicción de variables, 41

Características de la muestra, 43

Características de las intervenciones, 43

Discusión

CONCLUSIONES

REFERENCIAS 
$\mathrm{E}$ I asma es una enfermedad heterogénea multicausal caracterizada por la presencia de inflamación crónica, obstrucción del flujo aéreo, sensibilidad e hiperreactividad (Global Initiative for Asthma [GINA], 2016). Es un padecimiento complejo en el que interactúan factores predisponentes de carácter genético y desencadenantes ambientales que favorecen su desarrollo (Guía Mexicana de Asma [GUIMA], 2017).

En México, la prevalencia del asma oscila alrededor de los nueve millones de personas (García,et al, 2012; Roa, et al., 2009; Secretaría de Salud [SSA], 2016; Vargas, 2009). Durante el 2015 se ubicó en el cuarto lugar dentro de los padecimientos crónicos más atendidos en los servicios de salud, con un total de 226,803 casos, sólo por debajo de la obesidad, hipertensión arterial y diabetes mellitus tipo 2; siendo los adultos, de entre 22 y 44 años, quienes más solicitaron dicha atención (Dirección General de Epidemiología [DGE], 2015). Por su parte, en instituciones especializadas en padecimientos respiratorios, ocupa los primeros lugares de atención en consulta externa, urgencias y hospitalización (Instituto Nacional de Enfermedades Respiratorias [INER], 2012, 2014, 2016).

La continua atención del padecimiento en los servicios de salud se asocia con problemas para el control de la enfermedad. Dicha falta de control favorece repercusiones económicas, emocionales y de calidad de vida, además de incrementar el riesgo de crisis de asma, hospitalizaciones y muerte (Ebmeier, Thayabaran, Braithwaite, Bénamara, Weatherall y Beasley, 2017; Foster, Lavoie y Bouler, 2011; García et al., 2012; GINA, 2016; GUIMA; 2017).

Para mejorar el control de la enfermedad se ha determinado que la adherencia a los inhaladores de control es el pilar fundamental (Engelkes, Janssens, de Jongste, Sturkenboom y Verhamme, 2015; GINA, 2016). A este respecto, la adherencia a estos inhaladores oscila entre 30 y $70 \%$ (Bender y Bender, 2005; Fernández y Merino, 2015; Horne, 2006; Horne, 2011; Laforest, El Hasnaoui, et al, 2009), lo que refleja un problema porque un promedio de adherencia menor a $80 \%$ disminuye 14 veces la efectividad del tratamiento (Dunbar-Jacob, Schlenk y McCall, 2012).

Por tanto, es necesaria la identificación de factores reguladores de las conductas de adherencia. De manera general se han identificado los relacionados con la enfermedad y el tratamiento, sociodemográficos, interpersonales e individuales (Hotz, Kaptein, Pruitt, Sánchez-Sosa y Willey, 2003; Levesque, Li y Pahal, 2012; Palomino, Morales, Contreras y Salazar, 2015; Sánchez-Sosa, 2002), siendo estos últimos los que proporcionan mejores explicaciones y resultados de las conductas de adherencia (DiMatteo, 2004; Hotz et al., 2003; Leventhal, Weinman, Leventhal y Phillips, 2008; Sánchez-Sosa, 2002). Asimismo, los factores individuales implican pautas de comportamiento específicas, por lo que la adherencia se considera un fenómeno psicológico (Rodríguez-Campuzano y Rentería-Rodríguez, 2016); además, en ellos es necesario enfocar la investigación e intervención psicológica para mejorar la adherencia (Horne, 2011; Leventhal et al., 2008; Sánchez-Sosa, 1998).

En el caso del asma se ha comprobado que las causas más comunes para una baja adherencia son las creencias asociadas a la enfermedad y al tratamiento; por ejemplo, creer que no se necesita del inhalador de control, considerar que el inhalador generará importantes efectos adversos, que la enfermedad es episódica o cíclica, que el inhalador tiene un efecto limitado para el control de la enfermedad y que no se tiene control sobre la enfermedad, entre otras creencias (Bender y Bender, 2005; Foster, Lavoide y Boulet, 2011; Halm, Mora y Leventhal, 2006; Horne y Weinman, 2002; Horne, 2006).

El modelo de sentido común y representación de enfermedad [MSCRE] (Diefenbach y Leventhal, 1996) ha sido marco de referencia para abordar las creencias de los pacientes porque desde éste las conductas de adherencia se entienden como resultado de las representaciones individuales de la enfermedad y el tratamiento (Leventhal y Cameron, 1987; Leventhal, Breland, Mora y Leventhal, 2010). El concepto medular del MSCRE es la representación de la enfermedad, la cual se refiere a las creencias asociadas a la enfermedad, las cuales se estructuran en las dimensiones de identidad, causas, consecuencias, temporalidad, control, coherencia y representación emocional, y se pueden entender de la siguiente manera: identidad: hace referencia a la experiencia perceptual de la enfermedad, es decir, tipo, lugar y cantidad de síntomas o sensaciones somáticas que se consideren correspondientes a la enfermedad; temporalidad: se refiere a la duración que se cree que tendrá la enfermedad (aguda, crónica o cíclica); causas: se asocia con las creencias acerca de lo que causó la enfermedad y se pueden estudiar en términos de agentes externos (virus, bacterias, estrés cotidiano), susceptibilidad (edad, genética o herencia) y comportamientos (consumo de alcohol, tabaco y estilo de vida); consecuencias: se refiere a los efectos percibidos y experimentados en diferentes áreas (funcionamiento físico, psicológico y social, repercusiones económicas); control: hace referencia a las creencias respecto a 
la capacidad percibida de controlar la enfermedad y a las expectativas de qué tratamiento médico controle la enfermedad o disminuya los síntomas experimentados (control del tratamiento); coherencia: se relaciona con la claridad con que se entiende la enfermedad (tipo de síntomas, duración de los síntomas y temporalidad de la enfermedad), y representación emocional: dimensión que se refiere a las reacciones emocionales derivadas de los síntomas de la enfermedad, sensaciones somáticas experimentadas, o del resultado de vivir con un padecimiento crónico (Broadbent, Petrie, Main y Weinman, 2006; Cameron y Moss-Morris, 2004; Diefenbach y Leventhal, 1996; Mora y McAndrew, 2013; Moss-Morris, Weinman, Petrie, Horne, Cameron y Buick, 2002).

Desde hace algunos años, autores como Horne, Weinman y Hankins (1999), Horne y Weinman (2002) y Horne (2003) han propuesto la inclusión de la representación del tratamiento o Marco Necesidad-Preocupación [MNP] (Horne et al., 1999) y sus subdimensiones de necesidad y preocupación al MSCRE. De manera particular, la subdimensión de necesidad se puede entender en términos de la percepción que los pacientes tienen acerca de la necesidad de usar, en el caso del asma, el medicamento de control, y la preocupación implica la percepción de posibles efectos secundarios derivados de usar el medicamento de control. es necesario indicar que estas subdimensiones no se han incluido al MSCRE; sin embargo, Kucukarslan (2012), Aujla, Walker, Sprigg, Abrams, Massey y Vedhara (2016) y Unni y Shiyanbola (2016) lo consideran necesario para el trabajo de explicación, predicción e intervención en conducta de adherencia en personas con asma y otras enfermedades crónicas.

Hasta hoy, estudios de revisión y metaanálisis indican que no todas las subdimensiones del MSCRE predicen conductas de adherencia en personas con enfermedades crónicas (Aujla et al., 2016; Brandes y Mullan, 2014; Holmes, Hughes y Morrison, 2014; Kucukarslan, 2012). Explicaciones acerca de estos resultados se asocian con equiparar conductas de adherencia y conductas de automanejo, incluir una gran variedad de padecimientos (agudos y crónicos) y no incluir las dimensiones de necesidad y preocupación por el tratamiento (MNP). En contraparte, estudios de revisión y metaanálisis que han analizado la función predictiva de las dimensiones de necesidad y preocupación indican que son de las variables de mayor importancia (Dima, Hernandez, Cunillera, Ferrer, De Bruin y Grupo ASTRO-LAB, 2015; Foot, La Caze, Gujral y Cottrell, 2016; Holmes et al., 2014; Horne, Chapman, Parham, Freemantle, Forbes, Cooper, 2013).

Sobre todo en personas con asma, las revisiones han mostrado datos similares a los de los metaanálisis ya repor- tados (Kaptein, et al, 2008; Kaptein, Klok, Moss-Morris y Brand, 2010); sin embargo, en esta revisión se ha optado por incluir como variable dependiente al automanejo del asma y resultados clínicos en la enfermedad; además se han incluido estudios no derivados del MSCRE ni del MNP, en específico de intervención, y estudios con niños, jóvenes y adultos.

Por tanto, derivado de las consideraciones anteriores es necesaria una revisión actualizada de los estudios desde el MSCRE, incluidas las subdimensiones del MNP, que permita identificar las relaciones entre representación de la enfermedad, representación del tratamiento y conductas de adherencia a inhaladores de control en adultos con asma.

\section{Objetivo}

El propósito de este trabajo fue la identificación y descripción de estudios empíricos referentes a la relación entre representación de enfermedad, representación del tratamiento y adherencia a inhaladores de control en adultos con asma; derivados del MSCRE y el MNP.

\section{MÉTodo}

\section{Rango de la búsqueda}

Para la revisión narrativa el periodo de selección fue de 1999 a septiembre de 2018.

\section{Unidades de análisis}

Se utilizaron dos métodos; 1) se hizo una búsqueda en bases de datos electrónicas, las cuales fueron Scopus, Psiclnfo, MEDLINE, Scielo y Dialnet, y 2) una vez que se obtuvieron los estudios, se hizo una revisión en las referencias de éstos y la posterior búsqueda de estudios relevantes. Se utilizaron estas bases de datos debido a su gran importancia dentro de los recursos electrónicos de búsqueda en ciencias de la salud, medicina, psicología y ciencias del comportamiento, además de ser los recursos electrónicos más utilizados para la búsqueda de información especializada a nivel mundial (American Psychological Asssociation, 2018; Cañedo, Nodarse y Mulet, 2015).

\section{Núcleos temáticos}

Para la revisión en bases de datos se utilizaron las siguientes palabras clave: illness representation, illness perception, treatment representation, treatment perception, illness cognition, illness beliefs, treatment beliefs, medication beliefs, asthma, adherence y inhaled corticosteroids. 


\section{Artículos encontrados}

Los artículos encontrados fueron 272, de los cuales se hizo un análisis de sus características y pertinencia para incluirlos en la presente revisión narrativa. El proceso de análisis y selección se muestra en la figura 1.

\section{Composición de la muestra de artículos}

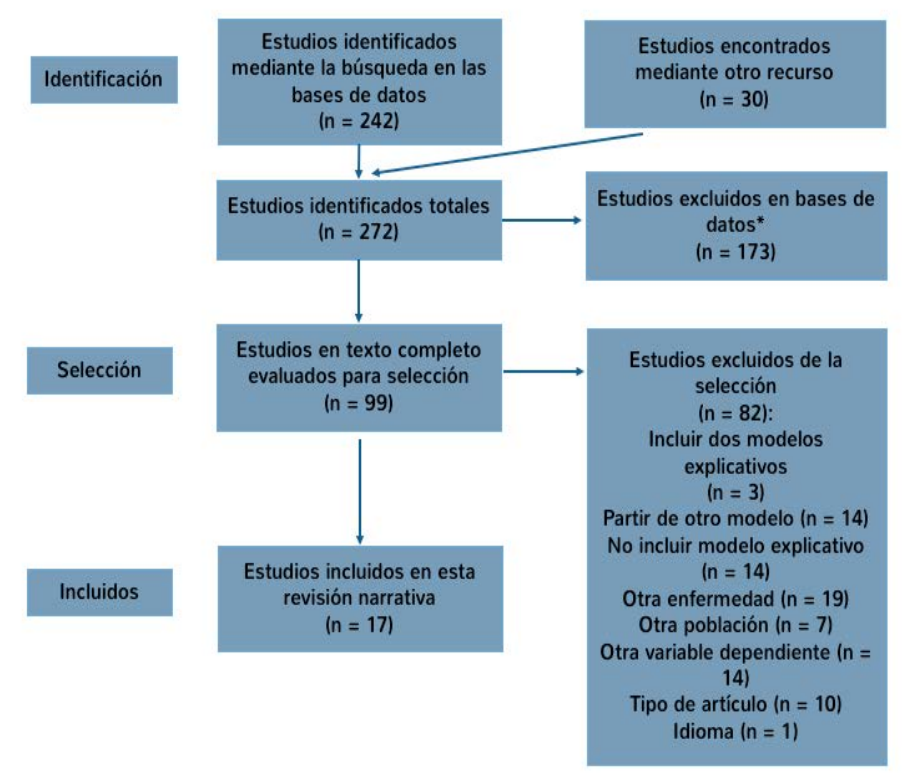

\section{Figura 1.}

Diagrama de identificación, selección e inclusión de estudios empíricos desde el MSCRE y las dimensiones de necesidad y preocupación, con base en las recomendaciones de PRISMA. * Los estudios excluidos durante la revisión de base de datos correspondieron a estudios que no se tuvo acceso a texto completo, aplicados en otra enfermedad y otros filtrados dentro de la búsqueda.

\section{Criterio de inclusión y exclusión de artículos}

Los estudios potencialmente seleccionables debían tener las siguientes características.

1. Tipos de estudio. Correlacionales, longitudinales, comparativos, experimentales y de intervención.

2. Participantes. Estudios hechos con adultos con diagnóstico confirmado de asma.

Objetivos: a) Relacionar las dimensiones de representación de enfermedad y/o representación del tratamiento con conductas de adherencia a inhaladores de control; identificar las dimensiones predictoras de representación de enfermedad y/o representación del tratamiento conductas de adherencia a inhaladores de control; b) comparar las conductas de adherencia a inhaladores de control con base en las dimensiones predictoras de representación de enfermedad y/o representación del tratamiento, y c) mejorar las conductas de adherencia a inhaladores de control con base en intervenciones que incluyan las dimensiones de representación de enfermedad y/o representación del tratamiento.

3. Resultados. Conductas de adherencia a inhaladores de control evaluadas de manera conductual (no resultados en enfermedad y control del asma).

4. Modelo teórico. Estudios desarrollados desde el MSCRE, además de incluir las dimensiones de la representación del tratamiento: necesidad y preocupación. No debían incluir algún otro modelo teórico para dar explicación a los resultados.

5. Idioma de los estudios. Los estudios podían ser en idioma inglés y en español.

6. Año de publicación. El periodo de selección fue de 1999 a septiembre de 2018.

\section{Procedimiento}

Las palabras clave que se indicaron en los núcleos temáticos se intercalaron unas con otras y se conjugaban con los operadores OR y AND; además, la búsqueda se filtró enfocándose en artículos de revistas, población de adultos, área de psicología y medicina e idioma inglés y español. El procedimiento para la descripción de la revisión se basó en algunas recomendaciones PRISMA para revisiones narrativas; por ejemplo, identificar el estudio como revisión en el título, la organización del resumen, justificación del estudio de revisión, incluir criterios de elegibilidad, fuentes de información, selección de estudios, inclusión del diagrama de flujo, características de los estudios y discusión de los resultados (Moher, Liberati, Tetzlaff, Altman, y Grupo Prisma, 2009).

\section{Resultados}

Los estudios incluidos en la presente revisión fueron 17 , mismos que se están divididos y organizados de la siguiente manera: 1) estudios transversales y longitudinales, cuyo propósito fue evaluar, correlacionar y predecir conductas de adherencia; 2) estudios experimentales para identificar las condiciones y variables asociadas con conductas de adherencia, y 3) estudios de intervención, que evalúan los efectos de programas para mejorar conductas de adherencia. Como se mencionó, todos los estudios partieron del MSCRE y las dimensiones de necesidad y preocupación como eje rector del estudio y la explicación de los resultados. 


\section{Características de los estudios}

\section{Estudios transversales y longitudinales}

En la tabla 1 se muestran los estudios transversales y longitudinales, los cuales suman 12; las características de éstos se muestran en ella.

\section{Características de la muestra}

Los estudios incluidos fueron publicados de 1999 a 2015. Todos los estudios fueron elaborados en inglés, de los cuales cinco (41.6\%) se desarrollaron en Estados Unidos, cinco (41.6\%) en Inglaterra y dos (16.6\%) en Holanda.

La población objetivo fueron adultos con asma, incluyendo a 3,845 pacientes; el estudio que menos participantes incluyó fue el de Byer y Myers (2000) con 64 pacientes; por lo contrario, el estudio de Cooper, Metcalf, Versnel, Upton, Walker y Horne. (2015) incluyó 1,757, siendo el que más participantes tuvo. Como se mencionó, la etapa etaria de los participantes en los estudios fue la adultez; sin embargo, el nivel de edad de éstos oscilaba entre 16 y 87 años; nueve estudios incluyeron exclusivamente a participantes adultos, tres consideraron a sujetos con 16 y 17 años, y la investigación de Sofianou, Martynenko, Wolf, Wisnivesky, Krauskopf, Wilson, Sanghavi, Leventhal, Halm y Federman (2013) fue dirigida de modo específico a adultos mayores (60-77 años).

Respecto al tiempo de evolución de la enfermedad y la severidad del asma, en siete estudios (58.3\%) no se reporta esta información; sin embargo, en los estudios donde lo refieren se identifica un rango que va de nueve meses a 78 años y un rango entre 10 y 30 años. Por su parte, sólo cuatro estudios (33.3\%) reportaron la severidad de asma; Ohm y Aaronson (2006) trabajaron con asma de leve a severa persistente y Halm et al. (2006), Ponieman, Wisnivesky, Leventhal, Musumeci-Szabó y Halm (2009) y Sofianou et al. (2013) se enfocaron en las categorías de mayor severidad (moderada y severa).

Referente a la estructura de los estudios expuestos, nueve $(75 \%)$ fueron de tipo correlacional transversal (cuatro con datos comparativos y cinco predictivos), dos $(12.5 \%)$ longitudinales (con evidencia predictiva) y uno (8.3\%) trasversal comparativo.

Las variables de análisis fueron la representación de enfermedad y la representación del tratamiento como variables independientes y las conductas de adherencia a los inhaladores de control como variable dependiente. Ambas representaciones fueron operacionalizadas en términos de preguntas específicas y de instrumentos válidos y confiables derivados del MSCRE (Mora y McAndrew, 2013). En 11 artículos (91.6\%) se utilizaron instrumentos para evaluar la representación de la enfermedad y la representación del tratamiento y las conductas de adherencia a inhaladores de control. Respecto a la representación de enfermedad, tres artículos (25\%) utilizaron el Illness Perception Questionnaire [IPQ] (Weinman, Petrie, Moss-Morris y Horne, 1996) o el Illness Perception Questionnaire-Revised [IPQ-R] (Moss-Morris et al., 2002), tres (25\%) el Brief Illness Perception Questionnaire [BIPQ] (Broadbent et al., 2006) y uno el Beliefs About Asthma Questionnaire [BAAQ] (Jessop y Rutter, 2003), en contraparte, tres estudios utilizaron preguntas específicas derivadas del MSCRE (25\%), principalmente de las dimensiones de identidad (no síntomas, no asma), temporalidad (crónica, cíclica y aguda) y control cura (expectativas de curación por el médico). Para la evaluación de la representación del tratamiento, el Beliefs about Medication Questionnaire [BMQ] (Horne et al., 1999) se utilizó en nueve investigaciones (75\%) y uno más empleó una pregunta de necesidad y otra de preocupación. Finalmente, la Medication Adherence Report Scale [MARS] (Horne y Hankins, 2002) fue el principal instrumento para evaluar conductas de adherencia en siete estudios (58.3\%), la escala breve de Morisky se utilizó en tres (25\%) y tres estudios utilizaron escalas diseñadas ad hoc (25\%).

El tipo de muestreo en todos los estudios fue no aleatorio y las formas de aplicación de los instrumentos en su mayoría fueron de manera presencial en siete estudios $(58.3 \%)$, seguidos por la aplicación vía correo electrónico con tres estudios (25\%), vía correo electrónico por medio de una página de internet en dos estudios (16.6\%) y vía correo postal en un estudio (8.3\%).

\section{Relación y predicción de las variables}

Las principales dimensiones del MSCRE que se asociaron con conductas de adherencia fueron necesidad del tratamiento, diferencia entre necesidad-preocupación (puntaje positivo), identidad, control (enfermedad y tratamiento), temporalidad (crónica) y consecuencias. Por lo contrario, las variables que correlacionan de manera negativa fueron la diferencia entre necesidad-preocupación (puntaje negativo), no necesidad, preocupación por efectos adversos, creencias de no síntomas no asma (no identidad y temporalidad cíclica y aguda), consecuencias, experiencias de efectos secundarios del tratamiento, pobre identidad, bajas consecuencias percibidas y percepción de limitada efectividad del tratamiento.

En esta línea los mejores predictores de adherencia a inhaladores de control fueron necesidad (con y sin síntomas de asma), preocupación, diferencia necesidad-preocupación, consecuencias, control, identidad y temporalidad (Byer y Myers, 2000; Cooper et al., 2015; Horne y Weinman, 1999; Horne y Weinman, 2002; 
Tabla 1.

Estudios trasversales y longitudinales de evaluación y relación entre representación de enfermedad, representación del tratamiento y adherencia a inhaladores de control en adultos con asma con base en el MSCRE

\section{ReFERENCIA}

\section{País}

N

Diseño

INSTRUMENTOS

Resultados

Adherencia correlacionó positivamente con necesidad $(r=0.28)$, con la diferencia necesidadHorne y Weinman Inglaterra 78 Correlacional
$(1999)^{*}$

$\begin{array}{lll}\begin{array}{l}\text { Byer y Myers } \\ (2000)\end{array} \quad \text { Inglaterra } 64 \quad \text { Correlacional } & \begin{array}{l}\text { IPQ, BMQ, } \\ \text { Escala Morisky } \\ \text { de adherencia }\end{array}\end{array}$
$\begin{aligned} & \text { Horne y Weinman } \quad \text { Inglaterra } 100 \text { Correlacional IPQ, BMQ, } \\ & \text { (2002) }\end{aligned}$ MARS

\begin{tabular}{|c|c|c|c|c|}
\hline $\begin{array}{l}\text { Jessop \& Rutter } \\
\text { (2003) }\end{array}$ & Inglaterra & 330 & Correlacional & $\begin{array}{l}\text { BAAQ, items } \\
\text { específicos } \\
\text { para } \\
\text { adherencia }\end{array}$ \\
\hline Halm et al. (2006) & $\begin{array}{l}\text { Estados } \\
\text { Unidos }\end{array}$ & 198 & Longitudinal & $\begin{array}{l}\text { Ítems } \\
\text { específicos } \\
\text { para } \\
\text { representación } \\
\text { de } \\
\text { enfermedad y } \\
\text { adherencia }\end{array}$ \\
\hline
\end{tabular}

$\begin{array}{llll}\text { Ohm y Aaronson } & \begin{array}{l}\text { Estados } \\ \text { Unidos }\end{array} & 97 \quad \text { Correlacional } & \begin{array}{l}\text { IPQ-R, PAS, } \\ \text { (2006) }\end{array}\end{array}$

Menckeberg et al. (2008)

Holanda

233

Correlacional

BMQ, MARS

$B M Q$, ítems específicos

Ponieman et al. Estados (2009) Estados
Unidos 261 preocupación ( $r=0.39$ ) y negativamente con preocupación $(r=-0.21)$. El mejor predictor de adherencia fue la diferencia necesidad-preocupación explicando $19 \%$ de la varianza.

Adherencia (número de inhalaciones) correlacionó con necesidad $(r=0.44)$, control $(r=0.32)$, temporalidad $(r=0.34)$ y causas $(r=0.36)$. Adherencia (autorreporte) correlacionó con necesidad $(r=0.44)$, identidad $(r=0.40)$ y temporalidad $(r=0.30)$. El principal predictor de adherencia fue necesidad, explicando $17.7 \%$ (número de inhalaciones) y $15.8 \%$ (autorreporte).

Adherencia correlacionó positivamente con necesidad $(r=0.32)$ y negativamente con consecuencias $(r=-0.24)$ y preocupación $(r=-0.43)$. Los mejores predictores de adherencia fueron necesidad y preocupación, que explicaron 38 y $31 \%$ respectivamente de la varianza de adherencia.

Adherencia se correlacionó positivamente y de manera débil con consecuencias $(r=0.18)$. En general la representación de enfermedad como predictor explica $\mathbf{2 8 . 7 \%}$ de la varianza de adherencia.

Temporalidad episódica se asoció con menor probabilidad de adherencia (OR $=4.49$ ), así como con la expectativa de cura $(O R=3.92)$. La temporalidad episódica reduce en un tercio la probabilidad de adherencia.

La identidad no se asoció con adherencia, esto al comparar un grupo de elevada identidad $(67 \%)$ y otro de baja identidad (34\%), no encontrándose diferencias estadísticamente significativas en el conteo de dosis $(t=86.93=-1.85)$, ni en el autorreporte $(t=92=-1.20)$.

Adherencia en autorreporte se asoció con necesidad $(r=0.35)$ y la diferencia necesidad-preocupación $(r=0.38)$. La adherencia medida por registros farmacéuticos se asoció con necesidad $(r=0.37)$ y la diferencia necesidad-preocupación $(r=0.32)$.

Se identificó que la percepción de necesidad de usar el medicamento, aunque no se tengan síntomas aumenta la probabilidad de adherencia (OR, 2.67.6). Por su parte, preocupación (OR, 0.4-0.5), percepción de dependencia del inhalador (OR, 0.4- para

representación

de enfermedad, MARS 0.6) y percepción de disminución de efectividad del inhalador $(O R, 0.4)$ aumentan la probabilidad de no adherencia. 
Temporalidad aguda, baja necesidad y mayor preocupación por el tratamiento se asociaron con

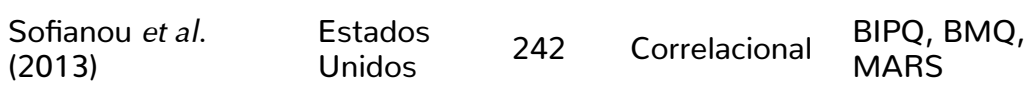
mayor probabilidad de baja adherencia. Por ello temporalidad aguda (OR $=0.45$ ), necesidad (OR $=0.91)$ y preocupación $(\mathrm{OR}=0.91)$ se asocian de modo diferencial con la probabilidad de una mejor adherencia.

$\begin{array}{llcll}\begin{array}{l}\text { Steenis et al. } \\ \text { (2014) }\end{array} & \text { Holanda } & 93 & \text { Correlacional } & \begin{array}{l}\text { BMQ, Escala } \\ \text { Morisky de } \\ \text { adherencia }\end{array} \\ \text { Cooper et al. (2015) } & \text { Inglaterra } & 1,757 & \text { Correlacional } & \text { BMQ, MARS }\end{array}$

Sólo necesidad de usar el medicamento correlacionó y se configuró como predictor de autorreporte de adherencia.

Sólo la preocupación por el uso del inhalador y efectos secundarios se asoció con baja adherencia ( $r$ $=0.46)$.

Pacientes con baja adherencia se caracterizaron con reportar una baja necesidad, elevada preocupación, baja percepción de síntomas, bajas consecuencias de la enfermedad y un bajo impacto emocional de ésta. Por lo contrario, pacientes adherentes se caracterizaron por reportar una elevada necesidad, baja preocupación, elevado control de la enfermedad y del tratamiento, elevada coherencia, baja consecuencias y bajo impacto emocional de la enfermedad.

Abreviaturas: r: Correlación de Pearson; t: Prueba t para grupos independientes, y OR: Odds Ratio o razón de momios. Instrumentos: BMQ: Beliefs about Medication Questionnaire; IPQ: IIIness Perception Questionnaire; IPQ-R: IIIness Perception Questionnaire-Revised; BIPQ: Brief IIIness Perception Questionnaire; BAAQ: Beliefs About Asthma Questionnaire, y MARS: Medication Adherence Report Scale y Escala de adherencia de Morisky. * En el estudio de Horne y Weinman (1999) también participaron 47 Enfermedad Renal Crónica en hemodiálisis, 116 cardiópatas y 83 con cáncer; sin embargo se incluyó en la revisión dada la relevancia del artículo.

Menckeberg, Bouvy, Bracke, Kaptein, Leufkens, Raaijmakers y Horne, 2008; Ponieman et al., 2009; Sofianou et al., 2013; Steenis, Driesenaar, Bensing, Van Hulteng, Souverein, Van Dijk, De Smet y Van Dulmen, 2014).

\section{Estudios experimentales}

Estos estudios se incluyeron porque cumplen con los criterios determinados y han evaluado hipótesis del modelo, resultados de investigación y proponen sugerencias para las futuras investigaciones y ampliaciones teóricas del MSCRE. En la tabla 2 se muestran los hallazgos de tres experimentos.

\section{Características de la muestra}

Se identificaron tres estudios experimentales relacionados con conductas de adherencia en adultos con asma y desarrollados con base en el MSCRE, los cuales se publicaron entre 2004 y 2014. Todos los estudios fueron escritos en inglés; dos se llevaron efectuaron en Estados Unidos y uno en Inglaterra.

Si bien la población objetivo de los estudios era adultos con asma, en los estudios de Kucukarslan, Lee, Patel y Ruparelia (2013) y Kucukarslan, Plumley, Chang

y Ueda (2014) todos los participantes fueron sujetos sanos y sólo Jessop et al. (2004) incluyeron a 36 personas con asma (GE); esto debido a la necesidad de tener datos de personas con poco tiempo con asma y con el uso del tratamiento. Los sujetos sin diagnóstico de asma (universitarios) fueron 282, de los cuales, en la investigación de Jessop, Rutter, Sharma y Albery (2004), 18 fungieron hipotéticamente como personas con asma y la muestra total de los estudios de Kucukarslan et al. (2013) y Kucukarslan et al. (2014) fue de 240. Es necesario indicar que en ningún estudio se reportó el promedio y nivel de edad para los participantes con asma, pero sí el de los participantes en general, esto en el estudio de Jessop et al. (2004) (rango = 17-55, media $(X)=22.9$, desviación estándar (DE) = 7.3). Acerca de los instrumentos de medición usados, dos estudios (66.6\%) utilizaron el IPQ-R para evaluar la representación de la enfermedad, en uno (33.3\%) usaron el BMQ para evaluar la representación del tratamiento y en dos $(66.6 \%)$ la MARS para evaluar adherencia.

\section{Relación y predicción de variables}

Los hallazgos más relevantes confirman datos de las investigaciones correlacionales y marcan precedente para 
Tabla 2.

Estudios experimentales acerca de representación de enfermedad, representación del tratamiento y adherencia a inhaladores de control en adultos con asma con base en el MSCRE

$\begin{array}{llll}\text { Referencia País N Instrumentos Procedimiento } & \text { Pesultados }\end{array}$

\begin{tabular}{|c|c|c|c|}
\hline $\begin{array}{l}\text { Jessop, } \\
\text { Rutter, } \\
\text { Sharma y } \\
\text { Alherv (2004) }\end{array}$ & Inglaterra & $\begin{array}{l}78 \\
\text { participantes, } \\
36 \text { con asma }\end{array}$ & $\begin{array}{l}\text { Ítems } \\
\text { específicos de } \\
\text { adherencia }\end{array}$ \\
\hline
\end{tabular}

Albery (2004)
Conformación del GE: estudiantes con asma; GC1: estudiantes sin asma; y GC2: estudiantes El tiempo de respuesta (interferencia) sin asma con lectura de experiencias de la enfermedad. El Gl y el GC1 eran expuestos a una tarea de Stroop con cuatro categorías de palabras (asma, síntomas de asma, palabras con contenido emocional y neutras) divididas en tres fases.

Por medio de una plataforma en línea, los participantes fueron asignados de manera aleatoria a escenarios hipotéticos (consecuencias elevadas o bajas, necesidad elevada o baja y preocupación elevada o baja); después leían la descripción y eran evaluados. fue mayor en el GE en palabras asociadas a asma y con contenido emocional. El nivel de adherencia se asoció con la interferencia en palabras asociadas a asma. Los participantes con baja adherencia mostraron mayor interferencia que aquellos con moderada adherencia.
Necesidad y consecuencias fueron predictores de satisfacción con el tratamiento. Consecuencias fue moderador y se asoció con necesidad y satisfacción del tratamiento. La satisfacción fue predictora de intención de adherencia, no así consecuencias, necesidad y preocupación.

$\begin{array}{llll}\begin{array}{l}\text { Kucukarslan, } \\ \text { Lee, Patel }\end{array} & \text { Estados } & 91 & \\ \text { y Ruperalia } & \text { Unidos } & \begin{array}{l}\text { universitarios } \\ \text { sanos }\end{array} & \text { BMQ, IPQ-R, } \\ \text { (2013) } & & \text { MARS }\end{array}$

Kucukarslan, Plumley, Chang, $y$ Ueda (2014) universitarios IPQ-R, MARS sanos
Por medio de una plataforma en línea, los participantes fueron asignados de modo aleatorio a los escenarios de percepción de la enfermedad. Cada sujeto recibió información (viñeta) de manera de percibir la enfermedad (combinación de identidad, consecuencias, causas y control personal) para su lectura; finalmente eran evaluados.

\author{
El control personal y las consecuencias \\ fueron los únicos predictores \\ significativos de intenciones de \\ adherirse. La intención de adherencia \\ en relación con los síntomas no fue \\ significativa porque los pacientes \\ no tienen experiencia con síntomas \\ y medicamentos controladores de \\ síntomas.
}

Abreviaturas: GE: Grupo Experimental; GC1: Grupo Control uno; GC2: Grupo Control dos. Instrumentos: BMQ: Beliefs about Medication Questionnaire; IPQ-R: IIIness Perception Questionnaire-Revised; MARS: Medication Adherence Report Scale. Diseños: Estudio 1: Cuasiexperimento con posprueba y grupo control; Estudio 2: Experimental factorial 2 × 2 × 2, y Estudio 3 : Experimental factorial $2 \times 2 \times 2 \times 2$.

el esclarecimiento del mantenimiento de conductas de adherencia en personas con asma. El primer hallazgo se asocia con la relación emocional entre experiencias sintomáticas de asma y las conductas de adherencia (Jessop et al., 2004). Se corrobora que la satisfacción con el tratamiento se relaciona con las conductas de adheren- cia y media la relación entre necesidad, preocupación y consecuencias de la enfermedad. Por último se comprueba que los principales predictores de adherencia a inhaladores de control son necesidad, consecuencias, satisfacción y control personal (Kucukarslan et al., 2013; Kucukarslan et al., 2014). 


\section{Estudios de intervención}

A diferencia de los estudios transversales y longitudinales, los estudios de intervención desde el MSCRE son escasos; en la tabla 3 se muestran los hallazgos de dos estudios de intervención para favorecer la adherencia a inhaladores de control en pacientes con asma desde el MSCRE.

\section{Características de la muestra}

Los dos estudios de intervención son recientes; fueron publicados entre 2012 y 2015; ambos estudios fueron escritos en inglés; uno se hizo en Nueva Zelanda y el otro en Inglaterra.

La población objetivo de los estudios fue adultos con asma, incluyendo a 278 pacientes. En el estudio de Petrie, Perry, Broadbent y Weinman (2012) se especificaron los criterios de inclusión para el estudio; el rango de edad de los participantes osciló entre 16 y 45 años, pero no se reportaron otras características sociodemográficas o clínicas. Por su parte, en el estudio de Chapman, Barnes, Barnes, Wilkinson, Hartley, Piddock, Weinman y Horne (2015) se especificaron los criterios de inclusión; la edad mínima de los participantes fue de 18 años y se reportaron características de la muestra como: promedio de edad (GC, $X=46.7, \mathrm{DE}=17.2 ; \mathrm{Gl}, \mathrm{X}=47.8, \mathrm{DE}=$ 16.6), años de educación (GC X $=19.5, \mathrm{DE}=8.74$; $\mathrm{GI} X$ $=18.6, \mathrm{DE}=8.43)$, tiempo de enfermedad $(\mathrm{GCX}=23.7$, $\mathrm{DE}=19.7 ; \mathrm{GI} X=22.1, \mathrm{DE}=19.7$ ), sexo (femenino: $\mathrm{GC}$ $\mathrm{n}=44$, GI n = 34), estado civil, ocupación, comorbilidades, consumo de tabaco e historia familiar de asma.

\section{Características de las intervenciones}

La estructura de la intervención fue similar en ambos estudios, esto es, evaluación, aleatorización a los grupos, intervención, post-evaluación y seguimiento. Las dos intervenciones utilizaron diseños experimentales.

Respecto a los instrumentos de medición usados en un estudio se empleó el BIPQ, representación de la enfermedad (Petrie et al., 2012), y en el segundo se utilizaron el BMQ (representación del tratamiento) y la MARS para evaluar conductas de adherencia (Chapman et al., 2015).

La intervención de Petrie et al. (2012) fue dirigida por psicólogos de la salud entrenados en el MSCRE, y el trabajo de Chapman et al. (2015) fue dirigido por enfermeras especialistas en asma. La intervención efectuada por Petrie et al. (2012) fue personalizada y estructurada con base en las dimensiones del MSCRE, es decir, el envío de mensajes de texto fungía como una estrategia informativa y de restructuración para modificar creencias asociadas a la enfermedad y el tratamiento, favoreciendo un cambio en el patrón de uso en el inhalador. Las creencias que se modificaron fueron la temporalidad (ten- dencia a la cronicidad), control de la enfermedad (mayor autoeficacia para su manejo) y aumento en la necesidad del tratamiento. En el mismo tenor, Chapman et al. (2015) usaron estrategias informativas y de discusión para identificar y modificar creencias de necesidad y preocupación acerca de los inhaladores de control; creencias en las que se generaron los principales cambios.

Respecto a la efectividad de las intervenciones, en el estudio de Petrie et al. (2012) se encontraron diferencias significativas entre grupos, tanto en la modificación de creencias, en el autorreporte de adherencia y en la proporción de paciente con una adherencia óptima. Por lo contrario, en el estudio de Chapman et al. (2015) se identificaron cambios en las creencias de necesidad y preocupación.

\section{Discusión}

Los resultados de esta revisión apoyan la importancia de los factores individuales, como las creencias respecto a la enfermedad y el tratamiento y su vínculo con las conductas de adherencia en los pacientes con asma, ya que, como se mencionó, estas últimas son la principal vía para el control del asma, por lo que es necesario incluir estos factores para favorecer este tipo de conductas (Horne, 2011; GINA, 2016; GUIMA, 2017).

Como se observó, las principales dimensiones del MSCRE, incluidas la necesidad y preocupación (MNP), que mejor se asocian y predicen conductas de adherencia a inhaladores de control fueron la necesidad del tratamiento, preocupación por efectos adversos del medicamento, diferencia necesidad-preocupación, control personal, temporalidad crónica y consecuencias, datos que concuerdan con revisiones previas (Dima et al., 2015; Foot et al., 2016; Holmes et al., 2014; Horne et al., 2013; Kaptein et al., 2008; Kaptein et al., 2010).

Por tanto, los programas dirigidos a promover adherencia al medicamento de control podrían incluir actividades para aumentar la percepción de necesidad del tratamiento, la autoeficacia para el manejo de la enfermedad, la percepción del asma como enfermedad crónica y no cíclica o episódica, además de abordar la disminución en la preocupación por efectos adversos del medicamento y las consecuencias percibidas de la enfermedad (Leventhal et al., 2010; McAndrew, Musumeci-Szabó, Mora, Vileikyte, Burns, Halm, Leventhal y Leventhal, 2008; Mora y McAndrew, 2013).

Respecto a los estudios experimentales, algunos datos a contrastar son la probable vinculación emocional entre las experiencias sintomáticas de asma y las conductas de adherencia (Jessop et al., 2004); sin embargo, en ningún estudio trasversal o longitudinal la representación 
Tabla 3.

Intervenciones para favorecer conductas de adherencia a inhaladores de control en adultos con asma desde el MSCRE

$\begin{array}{llll}\text { Referencia País } & \text { Intervención/ } & \text { Instrumentos } & \text { Procedimiento }\end{array}$

Tras una evaluación los participantes se asignaban de modo aleatorio al $\mathrm{Gl}$ $=57$ y al GTC = 46). La intervención consistió en un programa de mensajes de texto durante 18 semanas, mismos que se enviaron: 1) semana 1-6 dos mensajes diarios; 2) semana 7-12 un mensaje diario, y 3) semana 13-18 tres mensajes a la semana. Las post evaluaciones se hicieron al concluir la intervención y seis y nueve meses después.

Tras una evaluación (preconsulta) los participantes se asignaban aleatoriamente al Gl=57 y al GTC=79. La intervención consistió en una sesión informativa (consulta) y de discusión sobre adherencia y entrega de un manual educativo. En la postconsulta se evaluó la satisfacción con la consulta y un mes después se realizaba la

\section{posprueba.}

Se presentaron cambios en el GI entre el pre y post, en las creencias de temporalidad de la enfermedad, control de la enfermedad y creencia de necesidad. Durante todas las evaluaciones el promedio de adherencia fue mayor en el Gl que en el GC (t (122) = -3.06). La proporción de pacientes que aumentaron arriba de $80 \%$ de adherencia fue mayor en el GI ( 15 de $58 ; 25.9 \%$ ) y GC (7 de 66; $10,6 \%$ ) y la diferencia entre grupos fue de $15.3 \%$.
Chapman et al. (2015)
Inglaterra 136

BMQ, MARS
La posprueba mostró que el GI aumentó en necesidad del inhalador (t $(37)=2.25)$, redujeron su preocupación (t $(37=2.22)$ y aumentó la diferencia entre ambas (t $137=$ 2.81). No se identificaron cambios en adherencia entre GI y GC. Al final se identificó que los pacientes con baja adherencia creían menos necesario utilizar los medicamentos y tenían más preocupaciones por los efectos secundarios.

Abreviaturas: GI: Grupo de Intervención; GTC: Grupo de Tratamiento Convencional. Instrumentos: BMQ: Medication Adherence Report Scale; BIPQ: Medication Adherence Report Scale. Y MARS: Medication Adherence Report Scale. Diseño: Estudio 1: Experimental con preprueba-posprueba seguimiento y medidas repetidas y Estudio 2: Experimental con preprueba-posprueba y grupo control.

emocional correlacionó con las conductas de adherencia; de hecho esta variable no se incluyó en otros experimentos ni en las intervenciones. En este contexto los estudios sugieren que para determinar si hay o no relación entre la adherencia y los aspectos emocionales, la evaluación de estos últimos deberá ser asociada en particular con la enfermedad y no como una valoración genérica del estado emocional (Jessop y Rutter, 2003; Jessop et al., 2004; Kucukarslan, 2016; Unni y Shiyanbola, 2016).

Las evidencias sugieren que al evaluar e intervenir conductas de adherencia es necesario considerar siempre la preocupación por los efectos adversos del medicamento, dado que resulta una variable reguladora de este tipo de comportamientos. Por otro lado, las intervenciones hechas en etapas posteriores al diagnóstico de asma deberán enfocarse en el control personal para el manejo de consecuencias y adherencia a los inhaladores de control. En los experimentos se corroboró la relación entre necesidad, preocupación, consecuencias de la enfermedad y adherencia (Horne y Weinman, 2002) y la satisfacción con el tratamiento como mediador entre esta relación de variables. Por último, se comprueba que algunos de los principales predictores de adherencia a inhaladores de control son necesidad, consecuencias y control personal, datos derivados de los estudios observacionales (Byer y Myers, 2000; Cooper et al., 2015; Horne y Weinman, 1999; Horne y Weinman, 2002; Menckeberg et al., 2008; 
Ponieman et al., 2009; Sofianou et al., 2013; Steenis et al., 2014; Unni y Shiyanbola, 2016).

Respecto a los estudios de intervención, es evidente que los postulados teóricos del MSCRE y los resultados de los estudios transversales, longitudinales y experimentales no se han traducido en un número importante de estudios en pacientes con asma. Sin embargo, aunque los estudios de intervención identificados sólo son dos, se pueden retornar puntos de gran relevancia, como especificar los criterios de inclusión para los participantes, seleccionar a quienes se beneficiarán de las intervenciones (personas con problemas de adherencia, mayor severidad de enfermedad y que la representación de enfermedad y del tratamiento los pongan en riesgo de no ser adherentes), tener al menos dos sesiones de intervención y que pueden usarse diversas técnicas para el cambio conductual (Chapman et al., 2015; Leventhal et al., 2010; Petrie et al., 2012). Aunado a esto, en estos estudios las principales dimensiones del MSCRE y del MNP en que se intervino y en las que se generaron los principales efectos fueron en las creencias de temporalidad de la enfermedad (crónica), control de la enfermedad y creencia de necesidad, datos en concordancia con los estudios experimentales y observacionales.

A pesar de que en asma los estudios sean escasos, en otros padecimientos también hay evidencias de intervención desde el MSCRE y adherencia, como en infarto al miocardio (Broadbent, Ellis, Thomas, Gamble y Petrie, 2009; Yan, You, Liu, Jin, Zhou, Lin, Li y Gu, 2014), diabetes tipo II (Keogh, Smith, White, McGilloway, Kelly, Gibney y O'Dowd, 2011), enfermedad renal crónica (Karamanidou, Weinman y Horne, 2008), claudicación intermitente (Cunningham, Swanson, O'Caroll y Holdsworth, 2012), infección por VIH (Perera, Thomas, Moore, Faasse y Petrie, 2014) y artritis reumatoide (Zwikker, van den Ende, van Lankveld, den Broeder, van den Hoogen, van de Mosselaar, van Dulmen y van den Bemt, 2014). Es necesario indicar que la mayoría de las intervenciones tienen resultados positivos en la representación de enfermedad y conductas de adherencia; de este modo se puede comprobar en la revisión de intervenciones desarrollada por Jones, Smith y Llewellyn (2015), en la cual se incluyen varios de los estudios antes listados.

Una variable planteada de manera continua en la elaboración de este trabajo es el control del asma porque se le considera como el resultado principal de las conductas de adherencia a los medicamentos de control, es decir, los pacientes con mejor adherencia deberían estar mejor controlados. Sin embargo, no se consideraron estudios en los que se piensa el control del asma como una variable que resulta en el manejo de la enfermedad que también está vinculado con otras variables asociadas a la enfermedad y a cuestiones ambientales (Achstetter, Schultz, Faller y Schuler, 2016; GINA, 2016), además de no considerarse dentro de los objetivos de la revisión. A pesar de ello, también se ha desarrollado evidencia de la relación entre representación de enfermedad y control de la enfermedad (Achstetter et al., 2016; Bahçecioğlu y Çevikakyl, 2014; Lugo, Reynoso y Fernández-Vega, 2014).

Respecto al idioma de los estudios se consideraron en inglés y en español; de hecho, para la revisión en bases de datos se incluyeron dos que son exclusivamente para Latinoamérica; sin embargo no se encontraron estudios con características para ser incluidos en la revisión, y los que incluían las variables relevantes para este estudio se asociaban con el control del asma, ansiedad y depresión (Lugo et al., 2014) y estudios con niños y la representación de enfermedad de sus padres, mismos que fueron excluidos desde la revisión en base de datos.

En este contexto se plantea la necesidad de que para estudios futuros se incluya al control del asma como variable de resultado, y así identificar si los datos se relacionan entre representación de enfermedad, del tratamiento, conductas de adherencia y control del asma, o si hay resultados diferenciados al correlacionar estas formas de representación con adherencia y después con el control de la enfermedad.

Por otro lado, es evidente que la investigación desde estos modelos es limitada en el contexto latinoamericano, y por lo que dicen los resultados del trabajo desde estos modelos psicológicos en salud podrían aportar evidencias, variables relevantes de incluir en los programas de intervención y formas explicativas del cómo entienden su enfermedad, el tratamiento y las conductas de adherencia los pacientes con asma. Estos pocos estudios en adultos con asma promueven la posibilidad de hacer revisiones, pero en otro tipo de pacientes, ya sea en niños con asma y sus padres o en otro tipo de padecimientos.

\section{Conclusiones}

Los datos indican que el MSCRE es un modelo psicológico con aplicaciones importantes en el ámbito de la investigación, intervención y atención clínica de pacientes con asma. Así lo refieren también Horne, Price, Cleland, Costa, Covey, Gruffydd-Jones, Haughney, Hoegh, Kaplan, Langhammer, Østrem, Thomas, Van der Molen, Virchow y Williams (2007), Leventhal et al. (2010), Farmer (2012) y Kucukarslan (2016), quienes aseguran que al menos en el contexto clínico poco se trabaja con las representaciones y los modos de afrontamiento ante la enfermedad, por lo que el MSCRE funge como un mo- 
delo funcional y enfocado en el paciente para explicar y favorecer conductas de adherencia en pacientes con enfermedades crónicas, esto en virtud de que muchos de ellos tienden a ser no adherentes con base en sus primeras experiencias con los medicamentos, por lo que si éstas son negativas, las conductas de adherencia se verán comprometidas a lo largo de la enfermedad. En este sentido, los objetivos de las intervenciones, investigaciones o atención desde el MSCRE serían identificar las creencias que favorecer las conductas de adherencia, para así aumentar la eficacia de la farmacoterapia.

Lo anterior tiene sentido porque desde la perspectiva médica para mejorar la adherencia y el control del asma, la GINA (2016) considera a los programas de automanejo como una de las principales formas; sin embargo, dichos programas - a pesar de que son individualizados - parten sobre todo de la perspectiva del médico, no se basan en modelos explicativos del comportamiento y no están enfocados en el paciente, dejando de lado los siguientes aspectos: 1) la perspectiva de los pacientes acerca de los procedimientos a entrenar (identificación de desencadenantes, registro de síntomas, uso de inhaladores y flujómetro); 2) las creencias respecto a la enfermedad y el tratamiento (Horne, 2011; Horne et al., 2007; Østrem y Horne, 2015), y 3) causas individuales de falta de adherencia (Horne, 2006; Unni y Farris, 2011; Unni y Shiyanbola (2016).

En este contexto, Kucukarslan (2016) indica que explorar las creencias acerca de la enfermedad y el tratamiento ayuda a identificar las experiencias que los pacientes han tenido con su enfermedad, su vulnerabilidad y susceptibilidad percibidas. Por tanto, es necesario aceptar que la información que ofrece el equipo médico puede no corresponder con la de los pacientes, por lo que es necesario considerar las creencias de éstos y así orientar la toma de decisiones referente a adherencia de una manera más efectiva.

Lo descrito se ratifica en el hecho de que el médico indica a sus pacientes el uso diario del inhalador de control; para algunos no tendrá sentido usarlo; de este modo se ha identificado que los síntomas del asma vienen y van por temporadas, lo que favorecerá una concepción de la enfermedad como cíclica o aguda (Halm et al., 2006; Østrem y Horne, 2015). Por consiguiente, esta concepción ayudará a considerar que el inhalador no es necesario (Horne, 2006; Horne et al., 2013); que sólo es necesario cuando se tienen síntomas, y no lo es cuando no se presentan o no son perceptibles (Leventhal et al., 2012; McAndrew et al., 2008; Ponieman et al., 2009). Por tanto, si el equipo médico desconoce estos modos de entender la enfermedad, el tratamiento y su relación con las con- ductas de adherencia por parte de los pacientes se dejaría de lado variables de gran importancia, aspecto que se ha analizado de manera continua.

Se concluye con una serie de recomendaciones identificadas de los estudios revisados: 1) no evaluar adherencia en general en las personas con asma, sino específicamente la adherencia al medicamento de control; 2) hacer más estudios prospectivos para corroborar los resultados de las investigaciones transversales (Jessop y Rutter, 2003); 3) tener en cuenta el tiempo de evolución de la enfermedad y la severidad del asma, porque solo se reportó en cuatro estudios y podría haber diferencia en la percepción de enfermedad y del tratamiento con base en estas variables (Ponieman et al., 2009; Sofianou et al., 2013); 4) diferenciar el nivel de adherencia entre pacientes con baja necesidad y alta preocupación, alta necesidad y alta preocupación y baja preocupación y baja necesidad (subdimensiones del MNP) (Unni y Shiyanbola, 2016), y 5) trabajar con personas de reciente diagnóstico y con tiempo limitado usando los tratamientos, para así favorecer desde un inicio creencias funcionales respecto a la enfermedad y el tratamiento (Jessop et al., 2004; Jessop y Rutter, 2003; Kucukarslan, 2016; Kucukarslan et al., 2013; Kucukarslan et al., 2014).

\section{Referencias}

Achstetter, L. I., Schultz, K., Faller, H., \& Schuler, M. (2016). Leventhal's common-sense model and asthma control: Do illness representations predict success of an asthma rehabilitation?. Journal of Health Psychology, 1, 1-10. doi: 10.1177/1359105316651332

American Psychological Asssociation (APA, 2018). PsycINFO: A world-class resource for abstracts and citations of behavioral and social science research. Recuperado de: https://www.apa.org/pubs/databases/psycinfo/index.aspx

Aujla, N., Walker, M., Sprigg, N., Abrams, K., Massey, A., \& Vedhara, K. (2016). Can illness beliefs, from the commonsense model, prospectively predict adherence to selfmanagement behaviours? A systematic review and meta-analysis. Psychology \& Health, 31(8). 931-958. doi: 10.1080/08870446.2016.1153640

Bahçecioğlu, G., \& Çevikakyl, R. (2014). Determination of effect on asthma control of illness perception of asthma patients. Acta Medica Mediterránea, 30, 591-600. http:// www.actamedicamediterranea.com/

Bender, B. G., \& Bender, S. E. (2005). Patient-identified barriers to asthma treatment adherence: responses to interviews, focus groups, and questionnaires. Immunology and Allergy Clinics of North America, 25(1), 107-130. doi 10.1016/j.iac.2004.09.005

Brandes, K., \& Mullan, B. (2014). Can the common-sense model predict adherence in chronically ill patients? A metaanalysis. Health Psychology Review, 8(2), 129-153. doi:10. 
1080/17437199.2013.820986

Broadbent, E., Ellis, C.J., Thomas,J., Gamble, G., \& Petrie, K.J.(2009). Further development of an illness perception intervention for myocardial infarction patients: a randomized controlled trial. Journal of Psychosomatic Research, 67(1), 17-23. doi:10.1016/j.jpsychores.2008.12.001

Broadbent, E., Petrie, K., Main, J., \& Weinman, J. (2006). The brief illness perception questionnaire. Journal of Psychosomatic Research. 60, 631-637. doi:10.1016/j. jpsychores.2005.10.020

Byer, B., \& Myers, L. B. (2000). Psychological correlates of adherence to medication in asthma. Psychology, Health \& Medicine, 5(4), 389-393. doi: 10.1080/713690213

Cameron, P., \& Moss-Morris, R. (2004). Illness-related cognition and behavior. In: A. A., Kaptein, \& J. Weinman. Health Psychology (pp, 84-110), Oxford: BPS Blackwell.

Cañedo, A. R., Nodarse, R. M., \& Mulet, N. L. (2015). Similitudes y diferencias entre PubMed, Embase y Scopus. Revista Cubana de Información en Ciencias de la Salud, 26(1), 8491. http://www.redalyc.org/articulo.oa?id=377645760009

Chapman, S. C., Barnes, N., Barnes, M., Wilkinson, A., Hartley, J., Piddock, C., Weinman, J., \& Horne, R. (2015). Changing adherence-related beliefs about ICS maintenance treatment for asthma: feasibility study of an intervention delivered by asthma nurse specialists. BMJ open, 5(6), 1-9. doi: 10.1136/bmjopen-2014-007354

Cooper, V., Metcalf, L., Versnel, J., Upton, J., Walker, S., \& Horne, R. (2015). Patient-reported side effects, concerns and adherence to corticosteroid treatment for asthma, and comparison with physician estimates of side-effect prevalence: a UK-wide, cross-sectional study. Primary Care Respiratory Medicine, 25, 1-6. doi: 10.1038/ npjpcrm.2015.26.

Cunningham, M. A., Swanson, V., O'caroll, R. E., \& Holdsworth, R. J. (2012). Randomized clinical trial of a brief psychological intervention to increase walking in patients with intermittent claudication. British Journal of Surgery, 99(1), 49-56. doi: $10.1002 / \mathrm{bjs} .7714$

Diefenbach, M. A., \& Leventhal, H. (1996). The common-sense model of illness representation: Theoretical and practical considerations. Journal of Social Distress and the Homeless, 5(1), 11-38. doi: 10.1007/BF02090456

Dima, A. L., Hernandez, G., Cunillera, O., Ferrer, M., De Bruin, M. \& the ASTRO-LAB Group. (2015). Asthma inhaler adherence determinants in adults: Systematic review of observational data. European Respiratory Journal, 45, 994-1018. doi: 10.1183/09031936.00172114

DiMatteo, M. R. (2004). Variations in patients' adherence to medical recommendations: A quantitative review of 50 years of research. Medical Care, 42(3), 200-209. doi: 10.1097/01.mlr.0000114908.90348.f9

Dirección General de Epidemiología. (2015). Anuarios de morbilidad 1984-2015. Recuperado de http://www. epidemiologia.salud.gob.mx/anuario/html/anuarios.html

Dunbar-Jacob, J., Schlenk, E., \& McCall, M. (2012). Patient adherence to treatment regimen. En: A. Baum, T, A. Revenson \&J. Singer (eds) Handbook of Health Psychology (2ed) (pp. 271-292). Londres: Ed. Routledge. Taylor \& Francis Books.

Ebmeier, S., Thayabaran, D., Braithwaite, I., Bénamara, C.,
Weatherall, M., \& Beasley, R. (2017). Trends in international asthma mortality: analysis of data from the WHO Mortality Database from 46 countries (1993-2012). Lancet, 390, 935-45.

Engelkes, M., Janssens, H. M., de Jongste, J. C., Sturkenboom, M. C., \& Verhamme, K. M. (2015). Medication adherence and the risk of severe asthma exacerbations: a systematic review. European Respiratory Journal, 45(2), 396-407. doi: 10.1183/09031936.00075614

Farmer, K. C. (2012). Leventhal's common-sense model and medication adherence. Research in Social and Administrative Pharmacy, 8, 355-356. doi: 10.1016/j. sapharm.2012.05.013

Fernández, R. C., \& Merino, S. R. (2015). La adhesión a los tratamientos médicos: Concepto, medida y factores implicados. En: V. Plaza y C. Fernández (eds). Adhesión terapéutica en las enfermedades respiratorias. (pp 1-12). Barcelona: Viguera Editores, S.L.U. https://www. researchgate.net/publication/299126211

Foot, H., La Caze, A., Gujral, G., \& Cottrell, N. (2016). The necessityconcerns framework predicts adherence to medication in multiple illness conditions: A meta-analysis. Patient Education and Counseling, 99(5), 706-717. doi:10.1016/j. pec.2015.11.004

Foster, J. M., Lavoide, K. L., \& Boulet, L. P. (2011). Treatment adherence and psychosocial

factors in severe asthma. En: K. F. Chung, E. H. Bel \& S. E. Wenzel (eds) European Respiratory Monograph: Dificult to treat severe asthma, 51 (pp. 28-49). European Respiratory Society. doi: 10.1183/1025448x.erm5110

García, C., Fernández, R., Martínez, D., Franco, F., \& Pérez, J. (2012). Prevalencia y riesgos asociados con pacientes adultos con asma de 40 años o más de la Ciudad de México: Estudio de base poblacional. Salud Pública de México. 54, 425-432. PMID: 22832835

Global Initiative for Asthma. (2016). Global strategy for asthma management and prevention. Recuperado de www. ginasthma.org

Guía Mexicana de Asma. (2017). Guía Mexicana de Asma. Neumología y Cirugía de Tórax, 76, S1, 1-137.

Halm, E., Mora, P., \& Leventhal, H. (2006). No Symptoms, No Asthma. The acute episodic disease beliefs is associated with poor self-management among inner-city adults with persistent asthma. Chest, 129, 573-580. doi 10.1378/ chest.129.3.573

Holmes, E. A., Hughes, D. A., \& Morrison, V. L. (2014). Predicting adherence to medications using health psychology theories: a systematic review of 20 years of empirical research. Value in Health, 17(8), 863-876. doi: 10.1016/j. jval.2014.08.2671

Horne, R. (2003). Treatment perception and self-regulation. En: L. Cameron \& H. Leventhal (eds). The self-regulation of health and illness behaviour. (pp 138-154). London: Routledge, Taylor \& Francis Group.

Horne, R. (2006). Compliance, adherence, and concordance: Implications for asthma treatment. Chest Journal, 130(1), 65S-72S. doi: 10.1378/chest.130.1_suppl.65S

Horne, R. (2011). Adherence to asthma medication: A question of ability?. Primary Care Respiratory Journal, 20(2), 118119. doi: 118-119. DOI:10.4104/pcrj.2011.00054 
Horne, R., Chapman, S. C., Parham, R., Freemantle, N., Forbes, A., \& Cooper, V. (2013). Understanding patients' adherencerelated beliefs about medicines prescribed for long-term conditions: A meta-analytic review of the NecessityConcerns Framework. PloS one, 8(12), 1-24. doi:10.1371/ journal.pone. 0080633

Horne, R., \& Hankins, M. (2002). The Medication Adherence Report Scale. Brighton, England: Center for Health Care Research.

Horne, R., Price, D., Cleland, J., Costa, R., Covey, D., GruffyddJones, K., Haughney, J., Hoegh, S., Kaplan, A., Langhammer, A., Østrem, A., Thomas, M., Van der Molen, T., Virchow, J. C., \& Williams, S. (2007). Can asthma control be improved by understanding the patient's perspective?. BMC Pulmonary Medicine, 7(1), 1-11. doi: 10.1186/1471-2466-7-8

Horne, R., \&Weinman,J. (1999). Patients' beliefs about prescribed medicines and their role in adherence to treatment in chronic physical illness. Journal of Psychosomatic Research, 47(6), 555-567. doi: DOI: 10.1016/500223999(99)00057-4

Horne, R., \& Weinman, J. (2002). Self-regulation and selfmanagement in asthma: Exploring the role of illness perceptions and treatment beliefs in explaining nonadherence to preventer medication. Psychology \& Health, 17(1), 17-32. doi: $10.1080 / 08870440290001502$

Horne, R., Weinman. J., \& Hankins, M. (1999). The Beliefs about Medicines Questionnaire (BMQ): The development and evaluation of a new method for assessing the cognitive representation of medication. Psychology \& Health, 14(1), 1-24. doi: 10.1080/08870449908407311

Hotz, S., Kaptein, A., Pruitt, S., Sanchez-Sosa, J. J. \& Willey, C. (2003). Behavioural mechanisms explaining adherence: What every health professional should know. In: E. Sabate (ed.) Adherence to long term therapies: Evidence for action (pp. 135-149). Geneva: World Health Organization.

Instituto Nacional de Enfermedades Respiratorias. (2012). Informe anual 2011. Recuperado de www.iner.salud.gob.mx

Instituto Nacional de Enfermedades Respiratorias. (2014). Informe anual 2013. Recuperado de www.iner.salud.gob.mx

Instituto Nacional de Enfermedades Respiratorias. (2016). Informe anual 2015. Recuperado de www.iner.salud.gob.mx

Jessop, D. C., \& Rutter, D. R. (2003). Adherence to asthma medication: The role of illness representations. Psychology and Health, 18(5), 595-612. doi: 10.1080/0887044031000097009

Jessop, D. C., Rutter, D. R., Sharma, D., \& Albery, I. P. (2004). Emotion and adherence to treatment in people with asthma: An application of the emotional Stroop paradigm. British Journal of Psychology, 95(2), 127-147. doi: $10.1348 / 000712604773952386$

Jones, C. J., Smith, H. E., \& Llewellyn, C. D. (2015). A systematic review of the effectiveness of interventions using the Common Sense Self-Regulatory Model to improve adherence behaviours. Journal of Health Psychology, 21(11), 2709-2724. doi: 10.1177/1359105315583372

Kaptein, A. A., Hughes, B. M., Scharloo, M., Fischer, M. J., Snoei, L., Weinman, J., \& Rabe, K. F. (2008). Illness perceptions about asthma are determinants of outcome. Journal of Asthma, 45(6), 459-464. doi: 10.1080/02770900802040043

Kaptein, A., Klok, T., Moss-Morris, R., \& Brand, P. (2010).
Illness perceptions: Impact on self-management and control in asthma. Current Opinion in Allergy and Clinical Immunology, 10, 194-199. doi:10.1097/ ACl.0b013e32833950c1

Karamanidou, C., Weinman, J., \& Horne, R. (2008). Improving haemodialysis patients' understanding of phosphate-binding medication: A pilot study of a psycho-educational intervention designed to change patients' perceptions of the problem and treatment. British Journal of Health Psychology, 13(2), 205-214. doi:10.1348/135910708X288792

Keogh, K. M., Smith, S. M., White, P., McGilloway, S., Kelly, A., Gibney, J., \& O'Dowd, T. (2011). Psychological family intervention for poorly controlled type 2 diabetes. The American Journal of Managed Care, 17(2), 105-113. http:// www.ajmc.com/

Kucukarslan, S. N. (2012). A review of published studies of patients' illness perceptions and medication adherence: lessons learned and future directions. Research in Social and Administrative Pharmacy, 8(5), 371-382. doi:10.1016/j. sapharm.2011.09.002

Kucukarslan, S. N. (2016). Using the Common Sense Model in daily clinical practice for improving medication adherence. Journal of Clinical Outcomes Management, 23(5), 227230. www.jcomjournal.com

Kucukarslan, S. N., Lee, K. S., Patel, T. D., \& Ruparelia, B. (2013). An experiment using hypothetical patient scenarios in healthy subjects to evaluate the treatment satisfaction and medication adherence intention relationship. Health Expectations, 18(5), 1291-1298. doi: 10.1111/hex.12103

Kucukarslan, S., Plumley, D., Chang, A., \& Ueda, A. (2014). Intending to adhere or to not adhere: Results from an experiment in healthy subjects testing illness perceptions and behavioral intentions in asthma. Research in Social and Administrative Pharmacy, 10(1), 239-245. doi: http:// dx.doi.org/10.1016/j.sapharm.2013.05.001

Laforest, L., El Hasnaoui, A., Pribil, C., Ritleng, C., Osman, L. M., Schwalm, M. S., Le Jeunne, P., \& Van Ganse, E. (2009). Asthma patients' self-reported behaviours toward inhaled corticosteroids. Respiratory Medicine, 103(9), 1366-1375. doi:10.1016/j.rmed.2009.03.010

Leventhal, H., Breland, J. Y., Mora, H., \& Leventhal, E. (2010). Lay representations of Illness and treatment: A Framework for Action. In A. Steptoe (ed.), Handbook of Behavioral Medicine (pp, 137-154). USA: Springer Science-Business Media. doi 10.1007/978-0-387-09488-5_11

Leventhal, H., \& Cameron, L. (1987). Behavioral theories and the problem of compliance. Patient Education and Counseling, 10, 117-138. doi: 10.1016/0738-3991(87)90093-

Leventhal, H., Weinman, J., Leventhal, E., \& Phillips, A. (2008). Health Psychology: The search for pathways between behavior and health. Annual Review of Psychology. 59, 477-505. doi: 10.1146/annurev.psych.59.103006.093643

Levesque, A., Li, H. Z., \& Pahal, J. (2012). Factors related to patients' adherence to medication and lifestyle change recommendations: Data from Canada. International Journal of Psychological Studies, 4(2), 42-55. doi: 10.5539/ ijps.v4n2p42

Lugo, l., Reynoso, L., \& Fernández-Vega, M. (2014). Percepción 
de enfermedad, depresión, ansiedad y control del asma: Una primera aproximación. Neumología y Cirugía de Tórax. 73(2), 114-121. http://new.medigraphic.com/

McAndrew, L. M., Musumeci-Szabó, T. J., Mora, P. A., Vileikyte, L., Burns, E., Halm, E. A., Leventhal, E., \& Leventhal, H. (2008). Using the common sense model to design interventions for the prevention and management of chronic illness threats: from description to process. British Journal of Health Psychology, 13(2), 195-204. doi:10.1348/135910708X295604

Menckeberg, T. T., Bouvy, M. L., Bracke, M., Kaptein, A. A., Leufkens, H. G., Raaijmakers, J. A., \& Horne, R. (2008). Beliefs about medicines predict refill adherence to inhaled corticosteroids. Journal of Psychosomatic Research, 64(1), 47-54. doi:10.1016/j.jpsychores.2007.07.016

Moher, D., Liberati, A., Tetzlaff, J., Altman, D. G., \& Prisma Group. (2009). Preferred reporting items for systematic reviews and meta-analyses: The PRISMA statement. PLoS Medicine, 6(7), e1000097. doi: 10.1371/journal.pmed. 1000097

Mora, P., \& McAndrew. (2013). Common-sense model of self-regulation. En: M.D. Gellman \& J.R. Turner (eds.) Encyclopedia of Behavioral Medicine (pp. 460-467). New York: Springer. doi 10.1007/978-1-4419-1005-9

Moss-Morris, R., Weinman, J., Petrie, K., Horne, R., Cameron, L., \& Buick, D. (2002). The Revised Illness Perception Questionnaire (IPQ-R). Psychology \& Health. 17(1), 1-16. doi: $10.1080 / 08870440290001494$

Ohm, R., \& Aaronson, L. S. (2006). Symptom perception and adherence to asthma controller medications. Journal of Nursing Scholarship, 38(3), 292-297. doi: 10.1111/j.15475069.2006.00116.x

Østrem, A., \& Horne, R. (2015). Reducing asthma attacks: Consider patients' beliefs. Primary Care Respiratory Medicine, 25, 15021. doi:10.1038/npjpcrm.2015.21

Palomino, A. A., Morales, O. L., Contreras, J. O., \& Salazar, O. F. (2015). Cumplimiento terapéutico en asma: .Un problema de gran magnitud?. Iatreia, 28(4), 400-409. doi 10.17533/ udea.iatreia.v28n4a05.

Perera, A. I., Thomas, M. G., Moore, J. O., Faasse, K., \& Petrie, K. J. (2014). Effect of a smartphone application incorporating personalized health-related imagery on adherence to antiretroviral therapy: a randomized clinical trial. AIDS Patient Care and STDs, 28(11), 579-586. doi: 10.1089/apc.2014.0156

Petrie, K., Perry, K., Broadbent, E., \& Weinman, J. (2012). A text message programme designed to modify patients' illness and treatment beliefs improves self-reported adherence to asthma preventer medication. British Journal of Health Psychology, 17(1), 74-84. doi:10.1111/j.20448287.2011.02033.x

Ponieman, D., Wisnivesky, J. P., Leventhal, H., Musumeci-Szabó, T. J., \& Halm, E. A. (2009). Impact of positive and negative beliefs about inhaled corticosteroids on adherence in inner-city asthmatic patients. Annals of Allergy, Asthma \& Immunology, 103(1), 38-42. doi: $10.1016 / 51081-1206(10) 60141-X$

Roa, F., Toral, S., Roa, V., Zavala, J., Duran de Alba, L. M., Herrera, B \& Fuentes, F. (2009). Estimaciones sobre la tendencia del asma en México para el periodo 2008-2012. Anales Médicos, 54(1), 16-22. http://new.medigraphic.com/

Rodríguez-Campuzano, M. D. L., \& Rentería-Rodríguez, A.
(2016). Factores que impiden la adherencia a un régimen terapéutico en diabéticos: Un análisis descriptivo. Revista Psicología y Salud, 26(1). http://revistas.uv.mx/index.php/ psicysalud/article/view/1898

Sánchez-Sosa, J. J. (1998). Desde la prevención primaria hasta ayudar a bien morir: La interfaz intervención-investigación en psicología de la salud. En G. Rodríguez Ortega y M. E. Rojas Russell (coords) La Psicología de la salud en América Latina (pp. 33-44). México: M. A. Porrúa.

Sánchez-Sosa, J. J. (2002). Treatment adherence: The role of behavioral mechanisms and some implications for health care interventions. Revista Mexicana de Psicología. 19(1), 85-92. http://www.redalyc.org/revista.oa?id=2430

Secretaría de Salud. (SSA, 2016). Siete por ciento de la población en México padece asma. Recuperado de: https://www. gob.mx/salud/prensa/siete-por-ciento-de-la-poblacionen-mexico-padece-asma

Sofianou, A., Martynenko, M., Wolf, M., Wisnivesky, J., Krauskopf, K., Wilson, E., Sanghavi, M., Leventhal, H., Halm, M., \& Federman, A. (2013). Asthma beliefs are associated with medication adherence in older asthmatics. Journal of General Internal Medicine, 28(1), 67-73. doi: 10.1007/s11606-012-2160-z

Steenis, M. V, A., Driesenaar, J. A., Bensing, J. M., Van Hulteng, R., Souverein, P. C., Van Dijk, L., De Smet, P., \& Van Dulmen, A. M. (2014). Relationship between medication beliefs, selfreported and refill adherence, and symptoms in patients with asthma using inhaled corticosteroids. Patient Preference and Adherence. 8, 83-91. doi: 10.2147/PPA.S44185

Unni, E., \& Farris, K. B. (2011). Determinants of different types of medication non-adherence in cholesterol lowering and asthma maintenance medications: A theoretical approach. Patient Education and Counseling, 83(3), 382390. doi:10.1016/j.pec.2011.02.017

Unni, E., \& Shiyanbola, O. O. (2016). Clustering medication adherence behavior based on beliefs in medicines and illness perceptions in patients taking asthma maintenance medications. Current Medical Research and Opinion, 32(1), 113-121. doi: 10.1185/03007995.2015.1105204

Vargas, M. (2009). Epidemiología del asma. Neumología y Cirugía de Tórax, 68(S2), S91-S97. http://new.medigraphic.com/

Weinman, J., Petrie, K., Moss-Morris, R., \& Horne, R. (1996). The Illness Perception Questionnaire: A new method for assessing the cognitive representation of illness. Psychology \& Health, 11(1), 431-45. doi: 10.1080/08870449608400270

Yan, J., You, L. M., Liu, B. L., Jin, S. Y., Zhou, J. J., Lin, C. X., Li, Q., \& Gu, J. (2014). The effect of a telephone follow-up intervention on illness perception and lifestyle after myocardial infarction in China: A randomized controlled trial. International Journal of Nursing Studies, 51(6), 844855. doi: 10.1016/j.ijnurstu.2013.10.011

Zwikker, H. E., Van den Ende, C. H., Van Lankveld, W. G., Den Broeder, A. A., Van den Hoogen, F. H., Van de Mosselaar, B., Van Dulmen, S., \& Van den Bemt, B. J. (2014). Effectiveness of a group-based intervention to change medication beliefs and improve medication adherence in patients with rheumatoid arthritis: A randomized controlled trial. Patient Education and Counseling, 94(3), 356-361. doi: 10.1016/j.pec.2013.12.002 


\section{Meta-Análisis del Artículo}




\section{Dimensión Cuantitativa}

\section{Perfil de Evaluación entre pares}
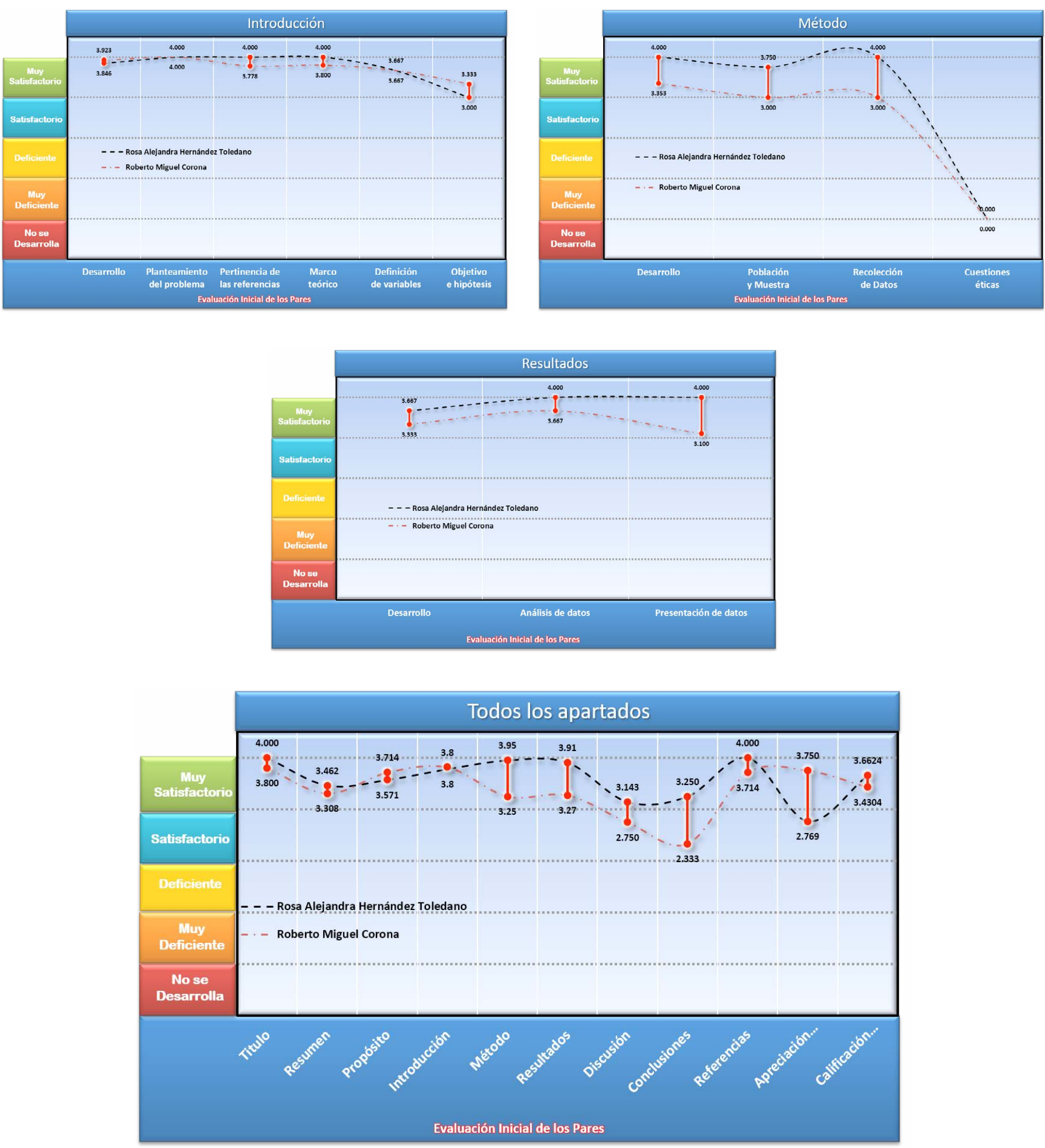


\section{Índice de Concordancia}

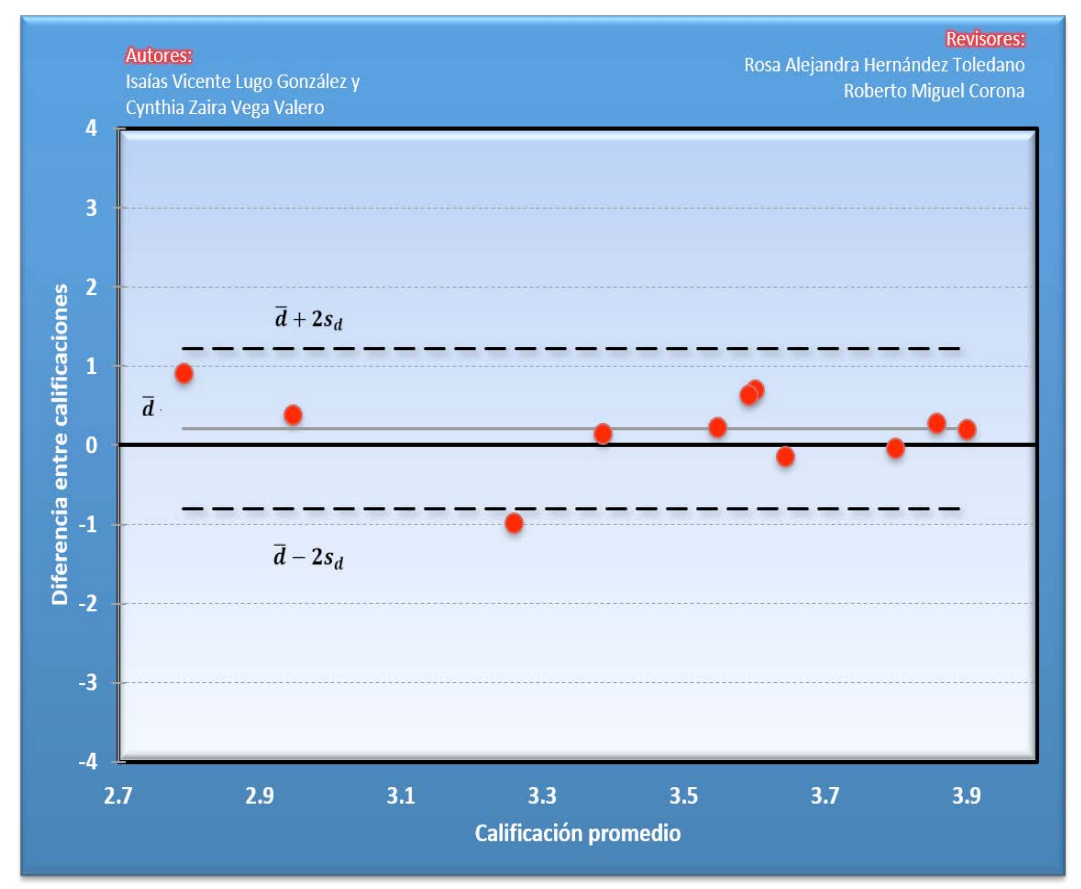

Índice de Acuerdo

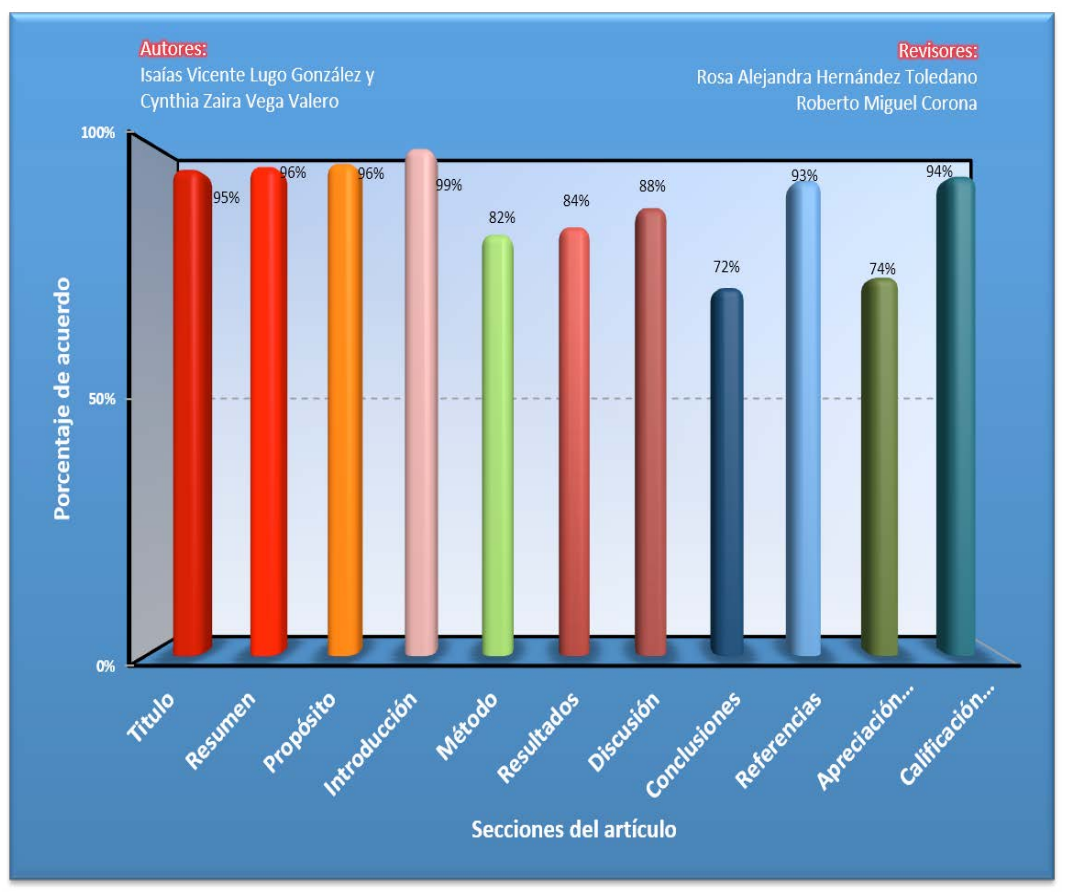




\begin{tabular}{|c|c|}
\hline Revisor 1 & Revisor 2 \\
\hline Rosa Alejandra Hernández Toledano & Roberto Miguel Corona \\
\hline \multicolumn{2}{|c|}{ Título/Autoría } \\
\hline Ninguno & $\begin{array}{l}\text { Con base en la redacción del título, es pertinente clarificar } \\
\text { la relación entre las variables ya que no es del toco claro } \\
\text { cuáles son las variables independientes y/o dependientes. }\end{array}$ \\
\hline \multicolumn{2}{|c|}{ Resumen } \\
\hline Ajustar el número de palabras al criterio de la revista. & $\begin{array}{l}\text { No cumple con el criterio de } 150 \text { palabras (definido por } \\
\text { la revista). }\end{array}$ \\
\hline \multicolumn{2}{|c|}{ Próposito del Estudio } \\
\hline Ninguno & es congruente en términos de una revisión de la literatura. \\
\hline \multicolumn{2}{|c|}{ Introducción } \\
\hline $\begin{array}{l}\text { El objetivo tiene que dar cuenta de los alcances de la } \\
\text { investigación, no de los procedimientos que se llevan a } \\
\text { cabo. El objetivo del trabajo debe expresar una guía del } \\
\text { estudio durante su desarrollo por lo que no pueden des- } \\
\text { cribir técnicas o acciones. Recomiendo acotar el aparta- } \\
\text { do a la identificación y descripción. Hacer uso de sinó- } \\
\text { nimos para evitar repetir palabras en el mismo párrafo }\end{array}$ & $\begin{array}{l}\text { Establecer una mejor conexión entre las variables del mo- } \\
\text { delo teórico y la importancia para la explicación de las va- } \\
\text { riaciones en la adherencia. }\end{array}$ \\
\hline \multicolumn{2}{|c|}{ Método } \\
\hline $\begin{array}{l}\text { Especificar que únicamente se utilizaron artículos } \\
\text { como unidades de análisis dentro de la búsqueda en } \\
\text { bases de datos. }\end{array}$ & $\begin{array}{l}\text { Falta describir los elementos del método PRISMA descri- } \\
\text { to en el procedimiento. }\end{array}$ \\
\hline
\end{tabular}




\begin{tabular}{|c|c|}
\hline Revisor 1 & Revisor 2 \\
\hline \multicolumn{2}{|c|}{ Resultados } \\
\hline $\begin{array}{l}\text { Los resultados se divide en tres grupos de estudio, para } \\
\text { cada caso se desarrolla un apartado de "Relación y pre- } \\
\text { dicción de variables", es necesario colocar las referencias } \\
\text { que permiten generar los hallazgos reportados. Así mis- } \\
\text { mo, la redacción de las características de las muestras } \\
\text { de los mismos tres grupos no permite hacer una lectu- } \\
\text { ra adecuada del contenido. En al apartado de estudios } \\
\text { transversales y longitudinales, se presentan únicamente } \\
\text { las siglas de los instrumentos encontrados. Dentro de los } \\
\text { estudios de intervención, el aparado de características de } \\
\text { las intervenciones es incoherente. Es necesario revisar la } \\
\text { redacción, faltas de ortografía, error de digitación, sig- } \\
\text { nos de puntuación y coherencia en el número (singular/ } \\
\text { plural), tanto en el apartado de resultados como en el } \\
\text { resto del manuscrito. Hacer uso de sinónimos para evi- } \\
\text { tar repetir palabras en el mismo párrafo. }\end{array}$ & $\begin{array}{l}\text { Resulta repetitiva la información redactada en el apartado } \\
\text { de los resultados y la incluida en las tablas. }\end{array}$ \\
\hline \multicolumn{2}{|c|}{ Discusión } \\
\hline $\begin{array}{l}\text { No se entiende la idea del primer párrafo, se consideran } \\
\text { varios puntos. La consideración del estudio de Horne y } \\
\text { Weinman podría ajustarse mejor en el método con los cri- } \\
\text { terios de inclusión. Es necesario revisar la redacción, faltas } \\
\text { de ortografía y coherencia en el número (singular/plural). } \\
\text { De igual manera se recomienda hacer uso de sinónimos } \\
\text { para evitar repetir palabras en el mismo párrafo. }\end{array}$ & $\begin{array}{l}\text { Uno de los puntos de discusión se centra en la carencia } \\
\text { de estudios de intervención basados en el modelo teórico } \\
\text { MSCRE y la población de interés; sin embargo, en el si- } \\
\text { guiente párrafo (p. 18) se argumenta que si es posible ob- } \\
\text { servar dichas intervenciones en otras poblaciones. Dichos } \\
\text { argumentos no se contrastan con profundidad en relación } \\
\text { a sus resultados con la VD y la relevancia con la población } \\
\text { de interés del presente escrito. }\end{array}$ \\
\hline \multicolumn{2}{|c|}{ Conclusiones } \\
\hline $\begin{array}{l}\text { La información proporcionada al final del segundo pá- } \\
\text { rrafo no concuerda con el objetivo del trabajo. Se re- } \\
\text { comienda hacer uso de sinónimos para evitar repetir } \\
\text { palabras en el mismo párrafo }\end{array}$ & $\begin{array}{l}\text { Con base en los lineamientos establecidos, la conclu- } \\
\text { sión no se ajusta a estos. Es pertinente para los auto- } \\
\text { res poder revisar los lineamientos de los apartados co- } \\
\text { rrespondientes a "Discusión" y "Conclusiones"; ya que } \\
\text { podría diferenciar de mejor manera los argumentos e } \\
\text { información contenida en cada uno. }\end{array}$ \\
\hline \multicolumn{2}{|c|}{ Referencias } \\
\hline Ninguna & $\begin{array}{l}\text { Algunas citas no coinciden con las referencias. Hay } \\
\text { errores en las referencias, correspondiente al uso de } \\
\text { "\&" La cita dentro del texto no incluye iniciales del } \\
\text { nombre de los autores. }\end{array}$ \\
\hline
\end{tabular}




\section{Historia del Proceso}

\section{EDITORIAL}

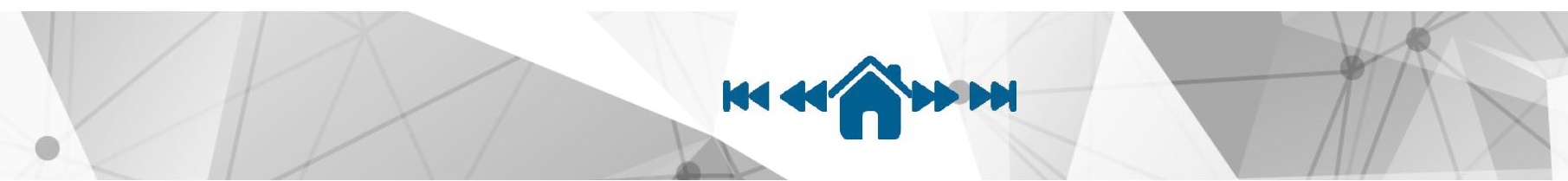

